\title{
A Study of the Asymptotic Equilibrium Behavior in Stratified Turbulence Submitted to Horizontal Shear
}

\author{
L. Thamri Naffouti ${ }^{1 \dagger}$, T. Lili $^{1}$ and M. Bouzaiane ${ }^{1,2}$ \\ ${ }^{1}$ Laboratoire de Mécanique des Fluides, Département de Physique, Faculté des Sciences de Tunis \\ ${ }^{2}$ Département de Physique, Faculté des Sciences de Bizerte \\ $\dagger$ Corresponding Author Email: thamrilamia@yahoo.fr
}

(Received December 12, 2012; accepted April 2, 2013)

\begin{abstract}
In this work, the asymptotic equilibrium behaviour of dimensionless parameters in stably stratified turbulence submitted to a horizontal shear is studied using two different methods. The first one is an analytic method and is based on linear solutions obtained when non linear effects of pressure and viscosity are neglected. The Laplace Transform is used for integrating differential system. The principal result of this first part of our work is the existence of asymptotic equilibrium states at high shear for all non dimensionless parameters. The second method is a numerical one and is based on a second-order modeling of equations. The Speziale Sarkar and Gatski (SSG) model is retained for pressure-strain correlation and dissipation time evolution equation, whereas, three of the most known second-order models are retained for the scalar field. The principal result of this second part is the big contribution of the SSG models for predicting asymptotic equilibrium states of non dimensional parameters.
\end{abstract}

Keywords: Stably stratified turbulence, Second order models, Asymptotic equilibrium behavior, Horizontal shear

\section{NOMENCLATURE}

$b \quad$ anisotropic tensor of Reynolds

$C_{p} \quad$ specific heat at constant pressure

g constant of gravity

$K \quad$ turbulent kinetic energy

$\mathrm{P}$ pressure

$\mathrm{p} \quad$ fluctuation of the pressure

$\mathrm{Ri}$ dimensionless Richardson number

$\mathrm{S}$ mean shear

$S_{\rho} \quad$ mean scalar gradient

$\bar{T}_{, i} \quad$ gradient of the scalar

$t$ time

$u_{i} \quad$ i-th component of the fluctuating velocity

$U_{i} \quad$ i-th component of mean velocity

$\overline{u_{i} u_{j}}$ reynolds stress tensor

$\overline{u_{i} \rho}$ turbulent flux of the scalar

$\bar{U}_{p, q}$ gradient of mean speed

$x_{i}$ component of an orthonormal Cartesian

coordinate system $\alpha \quad$ thermal diffusivity

$\mu \quad$ dynamic viscosity

$v \quad$ kinematic viscosity

$\lambda \quad$ viscosity ratio

$\tau \quad$ non dimensional time

$\delta_{i j} \quad$ Kronecker Symbol

$\rho$ fluctuation of the scalar

$\rho_{0}$ density of reference

$\overline{\rho^{2}}$ variance of scalar

$\varepsilon$ terms of dissipation of turbulent kinetic energy

$\varepsilon_{\rho \rho} \quad$ terms of dissipation of variance of the scalar

$\varepsilon_{i j} \quad$ terms of dissipation of tensor of Reynolds

$\varepsilon_{i \rho} \quad$ terms of dissipation of the scalar flux turbulent

$\varphi_{i j} \quad$ terms of pressure-strain correlation

$\varphi_{i} \rho$ terms of pressure- scalar gradient correlation 


\section{INTRODUCTION}

Turbulence phenomena are frequently met in the fluid flows and do not constitute an intrinsic property of the fluid. It is characterized using a whole of observation on the state of the movement. For example a turbulent flow tends to qualify a state of agitation of the movement where speeds move in a way apparently irregular, disordered and chaotic. In order to envisage the behavior of the turbulent flows, and for understanding well the complex turbulent processes of geophysics flows in the atmosphere and oceans, several authors (Holt et al. (1992) and Jacobitz et al. (1997) were interested to the analysis of different aspects of the coupled effects of a stable stratification (Bouzaiane et al. (2003b)) and a shear for a homogeneous turbulence. During the last years, Gerz et al. (1989) were interested to the study of the direct digital simulations for a homogeneous turbulence submitted to a vertical shear. They showed the influence of the Richardson number $\mathrm{Ri}$ on the turbulent parameters sizes. Komori et al. (1983) studied the case of a laminated flow in an opened water channel. Itsweire et al. (1988) showed the importance of the Richardson number $\mathrm{Ri}$ on the evolution of turbulence.

Turbulence in a vertically stably stratified fluid with uniform non vertical or horizontal shear has been considered in only a few investigations. Jacobitz and Sarkar (1998) performed a series of mean stream-wise velocity in which the angle $\theta$ between the gradients of mean density and mean streamwise velocity was varied from $\theta=0$ to $\theta=\frac{\Pi}{2}$. Laboratory experiments of turbulence in a stratified fluid with uniform horizontal shear have not been performed. However horizontal shear in experimental studies is present of fronts in a rotating stratified fluid (Chebbi et al. (2012)), stratified jets (Caldwell (1987)).

No many previous works have been interested to the horizontal shear except the result of DNS of Jacobitz and Sarkar (1999b) and Jacobitz (2002), this is surprising since the horizontal shear occurs frequently in environmental and many engineering applications. Examples are flow over topography, river in flow into the ocean or effluent discharge by power plants (Jacobitz (2002)). For our knowledge, no previous works has been dedicated to second-order modeling of the stably stratified turbulence submitted to a horizontal shear. Furthermor no coupling between SSG model and others models for scalar fields are known to authors. This constitutes the principal motivation of our work.

In section 2 equations of motion used in this study are introduced and the transports of second-order moments are derived. In section 3, analytical solutions in the case of high shear when non linear effects are neglected have been obtained. Solutions are investigated to study the asymptotic behavior at long time evolution of non dimensional parameters. The second-order modeling of transport equations of second moments makes the object of section 4 whereas their castling in non dimensional forms and numerical integration makes the object of section 5 . A peculiar attention will be accorded in this section also to the prediction of the asymptotic equilibrium states at long time evolution. Principal obtained results in this work, are summarized in section 6.

\section{MATHEMATICAL CONSIDERATIONS}

In an orthonormal Cartesian coordinate system of components $\left(\mathrm{x}_{1}, \mathrm{x}_{2}, \mathrm{x}_{3}\right)$, the flow to be considered in the present work is a two dimensional (2-D) homogeneous turbulent shear flow of a viscous incompressible fluid. The mean velocity $\bar{U}=\left(\bar{U}_{1}, 0,0\right)$ has a constant horizontal shear rate $\frac{\partial \bar{U}_{1}}{\partial x_{2}}=S$ (Jacobitz et al. (1999a)) whereas the scalar field presents a constant mean gradient $\frac{\partial \bar{\rho}}{\partial x_{3}}$.

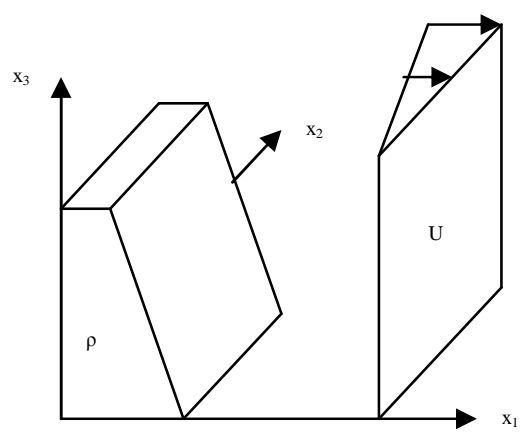

Fig. 1. Sketch of the mean density with vertical stratification and the mean velocity with horizontal shear

\subsection{Fundamental Equations}

The study of an incompressible turbulent shear flow is based on the continuity equation, the threedimensional unsteady Navier Stokes equation and a transport equation for the passive scalar. In the following, $x_{i}$ (with $i=1,2,3$ ) denotes the ith component of an orthonormal cartesian coordinate system. According to the classic Reynolds decomposition (Cadiou (1996a)), the dependent variables velocity $\tilde{U}_{i}$, density $\tilde{\rho}$ and pressure $\tilde{P}$ are decomposed into mean parts $\overline{U_{i}}, \bar{\rho}$ and $\bar{P}$ and a fluctuating parts $\mathrm{u}_{\mathrm{i}}, \rho$ and $\mathrm{p}$.

$\tilde{U}_{i}=\overline{U_{i}}+u_{i}, \tilde{\rho}=\bar{\rho}+\rho, \tilde{P}=\bar{P}+p$

The decomposition of the dependent variables is introduced into the equations of motion, and the following evolution equations for the fluctuating parts are obtained (Cadiou (1996b)):

$\frac{\partial u_{i}}{\partial x_{i}}=0$ 


$$
\begin{aligned}
& \frac{\partial u_{i}}{\partial t}+u_{k} \frac{\partial u_{i}}{\partial x_{k}}+S x_{2} \frac{\partial u_{i}}{\partial x_{1}}+S u_{2} \delta_{i 1}= \\
& -\frac{1}{\rho_{0}} \frac{\partial p}{\partial x_{i}}+v \frac{\partial^{2} u_{i}}{\partial x_{k} \partial x_{k}}-\frac{g}{\rho_{0}} \rho \delta_{i 3} \\
& \frac{\partial \rho}{\partial t}+u_{k} \frac{\partial \rho}{\partial x_{k}}+S x_{2} \frac{\partial \rho}{\partial x_{1}}+S_{\rho} u_{3}=\alpha \frac{\partial^{2} \rho}{\partial x_{k} \partial x_{k}}
\end{aligned}
$$

\subsection{Transport Equations}

In this section, transport equations for the components $\overline{u_{i} u_{j}}$ of the Reynolds stress, the components $\overline{u_{i} \rho}$ of the turbulent scalar flux, the variance of scalar $\overline{\rho^{2}}$ are obtained from basic Eqs. (1) to (3):

$\frac{d \overline{u_{i} u_{j}}}{d t}=P_{i j}-B_{i j}+\phi_{i j}-\varepsilon_{i j}$

Here $\frac{d}{d t}=\frac{\partial}{\partial t}+\overline{U_{k}} \frac{\partial}{\partial x_{k}}$ is the total time derivative.

$\frac{d \overline{u_{i} \rho}}{d t}=P_{i \rho}-B_{i \rho}+\phi_{i \rho}-\varepsilon_{i \rho}$

$\frac{d \overline{\rho^{2}}}{d t}=P_{\rho \rho}-2 \varepsilon_{\rho \rho}$

Where terms denoted by $\mathrm{P}$ are terms of production due to mean kinematic and scalar gradients:

$P_{i j}=-S \overline{u_{j} u_{2}} \delta_{i 1}-S \overline{u_{i} u_{2}} \delta_{j 1}$

$P_{i \rho}=-S \overline{u_{2} \rho} \delta_{i 1}-S_{\rho} \overline{u_{i} u_{3}}$

$P_{\rho \rho}=-2 S_{\rho} \overline{\rho u_{3}}$

Terms denoted by B are terms of gravity:

$B_{i j}=\frac{g}{\rho_{0}}\left(\overline{u_{i} \rho} \delta_{j 3}+\overline{u_{j} \rho} \delta_{i 3}\right)$

$B_{i \rho}=\frac{g}{\rho_{0}} \overline{\rho^{2}} \delta_{i 3}$

$\phi_{i j}$ and $\phi_{i \rho}$ are respectively terms of pressure-strain correlation and pressure-scalar gradient correlation: $\phi_{i j}=\frac{1}{\rho_{0}} \overline{p\left(\frac{\partial u_{i}}{\partial x_{j}}+\frac{\partial u_{j}}{\partial x_{i}}\right)} \quad \phi_{i \rho}=\frac{1}{\rho_{0}} \overline{p \frac{\partial \rho}{\partial x_{i}}}$

It is essential here to note that these last terms are the more complex terms to be modeled (Thamri et al. (2011) and Cadiou (1996a)).

Finally, terms $\varepsilon_{i j}, \varepsilon_{i \rho}$ and $\varepsilon_{\rho \rho}$ are terms of dissipation due to molecular effects:

$\varepsilon_{i j}=2 v \overline{\frac{\partial u_{i}}{\partial x_{k}} \frac{\partial u_{j}}{\partial x_{k}}}$

$\varepsilon_{i \rho}=(\alpha+v) \overline{\frac{\partial \rho}{\partial x_{k}} \frac{\partial u_{i}}{\partial x_{k}}}$

$\varepsilon_{\rho \rho}=-2 \alpha \overline{\frac{\partial \rho}{\partial x_{k}} \frac{\partial \rho}{\partial x_{k}}}$

While considering the trace of Eq.(4), we get the time evolution equation of turbulent kinetic energy $K=\frac{\overline{u_{i} u_{i}}}{2}$ $\frac{d K}{d t}=P-B-\varepsilon$

Where $P$ is the turbulent production term due tothe horizontal shear $\frac{\partial \overline{U_{1}}}{\partial x_{2}}$ :

$P=-S \overline{u_{1} u_{2}}$

B the buoyancy term:

$B=\frac{g}{\rho_{0}} \overline{u_{3} \rho}$

And $\varepsilon$ is the dissipation term due to molecular effects:

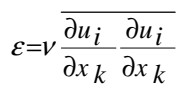

Classically, the potential energy $K_{\rho}$ is computed from the density fluctuations and is written as:

$K_{\rho}=\frac{1}{2} \frac{g}{\rho_{0} S_{\rho}} \overline{\rho^{2}}$

The equations for the components of the Reynolds stress $\overline{u_{1}^{2}}, \overline{u_{2}{ }^{2}}, \overline{u_{3}{ }^{2}}, \overline{u_{1} u_{2}}, \overline{u_{1} u_{3}}, \overline{u_{2} u_{3}}$, of the turbulent kinetic energy $K=\frac{\overline{u_{i} u_{i}}}{2}$, the components of the density flux $\overline{u_{1} \rho}, \overline{u_{2} \rho}, \overline{u_{3} \rho}$ and the equation of scalar variance $\overline{\rho^{2}}$, are consequently obtained.

Solutions of the obtained equations are now analysed firstly when non-linear effects of viscosity and pressure according to the DNS results of Holt et al. (1992), are neglected at high shear. This will be detailed in the following sub-section.

\subsection{Linear Solutions at High Shear}

Holt et al. (1992), non linear effects of viscosity and pressure have negligible effects at high shear (Holt et al. (1992) and Bouzaiane et al. (2003a)). If we take into account this result in the transport Eqs.(4), (5) and (6), terms denoted $\varphi$ and $\varepsilon$ are neglected. We obtain a system of ten first order coupled linear differential equations.

$$
\begin{aligned}
& \frac{d \overline{u^{2}}}{d t}=-2 S \overline{u_{1} u_{2}} \\
& \frac{d \overline{u_{2}^{2}}}{d t}=0 \\
& \frac{d \overline{u^{2}}}{d t}=-2 \frac{g}{\rho_{0}} \overline{u_{3} \rho} \\
& \frac{d \overline{u_{1} u_{2}}}{d t}=-S \overline{u_{2}^{2}}
\end{aligned}
$$


L. Naffouti et al. / JAFM, Vol. 7, No. 3, pp. 401-413, 2014.

$$
\begin{aligned}
& \frac{d \overline{u_{1} u_{3}}}{d t}=-S \overline{u_{2} u_{3}}-\frac{g}{\rho_{0}} \overline{u_{1} \rho} \\
& \frac{d \overline{u_{2} u_{3}}}{d t}=-\frac{g}{\rho_{0}} \overline{u_{2} \rho} \\
& \frac{d K}{d t}=-S \overline{u_{1} u_{2}}-\frac{g}{\rho_{0}} \overline{u_{3} \rho} \\
& \frac{d \overline{u_{1} \rho}}{d t}=-S \overline{u_{2} \rho}-S \rho^{u_{1} u_{3}} \\
& \frac{d \overline{u_{2} \rho}}{d t}=-S \frac{\rho_{2} u_{3}}{d \overline{u_{3} \rho}}=-S_{\rho} \overline{u_{3}^{2}}-\frac{g}{\rho_{0}} \overline{\rho^{2}} \\
& \frac{d \overline{\rho^{2}}}{d t}=-2 S_{\rho} \overline{u_{3} \rho}
\end{aligned}
$$

In a previous work, Bouzaiane et al. (2003c) have proposed linear solution for homogeneous sheared turbulence submitted to rotation. The same method used by Bouzaiane et al. (2003c) and Chebbi el al. (2012) is used in the present work.

We investigate solutions of Eqs.(4), (5) and (6) when non-linear effects are neglected.

Laplace Transform of a function $\mathrm{f}$, defined for a position $\mathrm{x}$ by: $L(f(x))_{S}=\int_{0}^{\infty} f(x) e^{-s x} d x$ is used and following solutions parameterized by the gradient Richardson number $\mathrm{Ri}(\mathrm{Ri}>0)$ are obtained:

$$
\begin{aligned}
& \overline{u_{1}^{2}}(\tau)=C_{11}+A_{11} \tau+B_{11} \tau^{2}+D_{11} C h\left(\sqrt{R_{i}} \tau\right)+ \\
& E_{11} S h\left(\sqrt{R_{i}} \tau\right)+F_{11} S h\left(2 \sqrt{R_{i}} \tau\right) \\
& \overline{u_{2}^{2}}(\tau)=C_{22} \\
& \overline{u_{3}^{2}}(\tau)=C_{33}+F_{33} S h\left(2 \sqrt{R_{i}} \tau\right)+K_{33} C h\left(2 \sqrt{R_{i}} \tau\right) \\
& \overline{u_{1} u_{2}}(\tau)=C_{12}+A_{12} \tau+B_{12} C h\left(\sqrt{R_{i}} \tau\right) \\
& \overline{u_{1} u_{3}}(\tau)=C_{13}+A_{13} \tau+B_{13} C h\left(\sqrt{R_{i}} \tau\right)+ \\
& D_{13} S h\left(\sqrt{R_{i}} \tau\right)+E_{13} \tau C h\left(\sqrt{R_{i}} \tau\right)+ \\
& F_{13} S h\left(\sqrt{R_{i}} \tau\right)+K_{13} C h\left(2 \sqrt{R_{i}} \tau\right) \\
& \overline{u_{2} u_{3}}(\tau)=C_{23}+A_{23} C h\left(\sqrt{R_{i}} \tau\right)+B_{23} S h\left(\sqrt{R_{i}} \tau\right) \\
& \overline{u_{1} \rho}(\tau)=C_{1 \rho}+A_{1 \rho} C h\left(\sqrt{R_{i}} \tau\right)+ \\
& B_{1 \rho} S h\left(\sqrt{R_{i}} \tau\right)+D_{1 \rho} \tau S h\left(\sqrt{R_{i}} \tau\right)+ \\
& E_{1 \rho} C h\left(2 \sqrt{R_{i}} \tau\right)+F_{1 \rho} S h\left(2 \sqrt{R_{i}} \tau\right) \\
&
\end{aligned}
$$

$$
\begin{aligned}
& \overline{u_{2} \rho}(\tau)=A_{2 \rho} C h\left(\sqrt{R_{i}} \tau\right)+B_{2 \rho} \operatorname{Sh}\left(\sqrt{R_{i}} \tau\right) \\
& \overline{u_{3} \rho}(\tau)=A_{3 \rho} \operatorname{Ch}\left(2 \sqrt{R_{i}} \tau\right)+B_{3 \rho} \operatorname{Sh}\left(2 \sqrt{R_{i}} \tau\right) \\
& \overline{\rho^{2}}(\tau)=C_{\rho}+A_{\rho} \operatorname{Ch}\left(2 \sqrt{R_{i}} \tau\right)+B_{\rho} \operatorname{Sh}\left(2 \sqrt{R_{i}} \tau\right) \\
& \overline{q^{2}}=\overline{u_{1}^{2}}+\overline{u_{2}^{2}}+\overline{u_{3}^{2}} \\
& \overline{q^{2}}=C_{q}+A_{q} \tau+B_{q} \tau^{2}+D_{q} C h\left(\sqrt{R_{i}} \tau\right)+ \\
& E_{q} \operatorname{Sh}\left(\sqrt{R_{i}} \tau\right)+F_{q} C h\left(2 \sqrt{R_{i}} \tau\right)+H_{q} \operatorname{Sh}\left(2 \sqrt{R_{i}} \tau\right)
\end{aligned}
$$

Here the coefficients A, B, C, D, E, F, H and K (see appendix A for detailed forms of these coefficients) are functions of initial conditions of turbulent parameters and Richardson number $R_{i}=\frac{g}{\rho_{0}} \frac{S_{\rho}}{S^{2}}=\frac{N^{2}}{S^{2}}$ (Rohr et al. (1988)), where $\mathrm{S}$ is the shear rate, $N$ the Brunt-Vaisala frequency $N^{2}=-\frac{g S_{\rho}}{\rho_{0}}$ and $\tau=S t$ is the non dimensional time. The analytic solutions will now be investigated to study the asymptotic behaviour of dimensionless parameters at long time evolution. This will make the object of the following subsection.

\subsection{Asymptotic Behaviour of Non Dimensional Parameters}

We begin by the study of the asymptotics behavior of non dimensional kinematic parameters, which are the components $b_{11}, b_{22}, b_{33}, b_{12}, b_{13}$ and $b_{23}$ of the anisotropy tensor of Reynolds $\left(b_{i j}=\frac{u_{i} u}{2 K}-\frac{1}{3} \delta_{i j}\right)$. After we extended, our study to the scalar dimensionless parameters, namely the ratios of buoyancy to production term $\frac{B}{P}$, the ratio of the potential energy to kinetic energy $\eta=\frac{1}{2} \frac{g}{\rho_{0} S_{\rho}} \frac{\overline{\rho^{2}}}{k}=\frac{k \rho}{k}, \frac{\overline{u_{1} \rho}}{\overline{u_{2} \rho}}$ and the correlation coefficient $\frac{\overline{u_{1} \rho}}{u_{1}^{\prime} \rho^{\prime}}$ (Where $u_{1}^{\prime}=\sqrt{\overline{u_{1}^{2}}}, \rho^{\prime}=\sqrt{\overline{\rho^{2}}}$ ). Expressions of all dimensional parameters are easily deduced from the above solutions.

At long time evolution, corresponding to $\tau=S t \rightarrow \infty$, solutions (32-42) lead to the simple relation of dimensionless parameters:

$\left(b_{11}\right)_{\infty}=\lim _{\tau \rightarrow \infty}\left(\frac{\overline{u_{1}^{2}}}{\overline{q^{2}}}-\frac{1}{3}\right)=\frac{F_{11}}{F_{q}+H_{q}}-\frac{1}{3}$ 


$$
\begin{aligned}
& \left(b_{22}\right)_{\infty}=\lim _{\tau \rightarrow \infty}\left(\frac{\overline{u_{2}^{2}}}{q^{2}}-\frac{1}{3}\right)=-\frac{1}{3} \\
& \left(b_{33}\right)_{\infty}=\lim _{\tau \rightarrow \infty}\left(\frac{\overline{u_{3}^{2}}}{\overline{q^{2}}}-\frac{1}{3}\right)=\frac{F_{33}+K_{33}}{F_{q}+H_{q}}-\frac{1}{3} \\
& \left(b_{12}\right)_{\infty}=\lim _{\tau \rightarrow \infty}\left(\frac{\overline{u_{1} u_{2}}}{q^{2}}\right)=0 \\
& \left(b_{13}\right)_{\infty}=\lim _{\tau \rightarrow \infty}\left(\overline{\frac{u_{1} u_{3}}{\overline{q^{2}}}}\right)=\frac{K_{13}}{F_{q}+H_{q}} \\
& \left(b_{23}\right)_{\infty}=\lim _{\tau \rightarrow \infty} \frac{\overline{u_{2} 2_{3}}}{\overline{q^{2}}}=0 \\
& \left(\frac{B}{P}\right)_{\infty}=\lim _{\tau \rightarrow \infty}\left(\frac{g}{\rho_{0}} \frac{\overline{u_{3} \rho}}{\overline{u_{1} u_{3} S}}\right)=\frac{g}{\rho_{0} S} \frac{A_{3 \rho}+B_{3 \rho}}{K_{13}} \\
& (\eta)_{\infty}=\lim _{\tau \rightarrow \infty}\left(\frac{g}{\rho_{0}} \frac{\overline{\rho^{2}}}{\overline{q^{2}}}\right)=\frac{g}{\rho_{0}} \frac{A \rho+B \rho}{F_{q}+H_{q}} \\
& \left(\overline{\frac{u_{1} \rho}{u_{2} \rho}}\right)_{\infty}=\lim _{\tau \rightarrow \infty}\left(\overline{\frac{u_{1} \rho}{u_{2} \rho}}\right)=\frac{E_{1 \rho}+F_{1 \rho}}{A_{2 \rho}+B_{2 \rho}} \\
& \left(\frac{\overline{u_{1} \rho}}{u_{1}^{\prime} \rho^{\prime}}\right)_{\infty}=\lim _{\tau \rightarrow \infty}\left(\frac{\overline{u_{1} \rho}}{\bar{u}_{1}^{\frac{1}{2}} \frac{1}{\rho^{2}}}\right)=\frac{E_{1 \rho}+F_{1 \rho}}{F_{11}^{\frac{1}{2}}\left[A_{\rho}+B \rho\right]^{\frac{1}{2}}}
\end{aligned}
$$

The obtained expressions $\left(b_{11}\right)_{\infty},\left(b_{22}\right)_{\infty}$, $\left(b_{33}\right)_{\infty},\left(b_{12}\right)_{\infty},\left(b_{13}\right)_{\infty},\left(b_{23}\right)_{\infty},\left(\frac{B}{P}\right)_{\infty}$, $(\eta)_{\infty},\left(\overline{\frac{\overline{u_{1} \rho}}{\overline{u_{2} \rho}}}\right)_{\infty}$ and $\left(\overline{\frac{u_{1} \rho}{u_{1} \rho}}\right)_{\infty}$ are only functions of constant coefficients A, B, F, H, K and E presented in appendix A. These solutions confirm the existence of an asymptotic equilibrium behavior at long time evolution of dimensionless parameters (Ben Abdallah et al. (2005)) $\mathrm{b}_{11}, \mathrm{~b}_{22}, \mathrm{~b}_{33}, \mathrm{~b}_{12}, \mathrm{~b}_{13}$, $\mathrm{b}_{23}, \frac{B}{P}, \frac{\overline{u_{1} \rho}}{\overline{u_{2} \rho}}, \frac{\overline{u_{1} \rho}}{u_{1} \rho}$ and $\eta=\frac{E \rho}{k}$. Furthermore, they show that these states are only functions of the Richardson number $\mathrm{Ri}$ and the initial values of dimensionless parameters. This first approach is only a qualitative one. A quantitative analysis of the behaviour of dimensionless turbulent parameters, based on a second-order modeling of the behaviour of dimensionless parameters will be developed in the following sections.

\section{SECOND-ORDER MODELING FOR DIMENSIONAL EQUATIONS}

Second-order modeling remains one of the more important approaches to understand and study geophysical turbulent flows and complex configurations of turbulent flows (Khaleghi et al. (2010)). In the following sub-section, a brief introduction to second-order modeling followed by the principal second-order models is presented.

\subsection{Second-Order Modeling}

In this part, second-order turbulence closure models are retained to close transport Eqs.(21) to (31). The pressure-strain correlation $\phi_{i j}$ and the pressurescalar gradient correlation $\phi_{i \rho}$ are the principal terms to be modeled in evolution equations of Reynolds stress and turbulent scalar flux. These correlations $\phi_{i j}$ and $\phi_{i \rho}$ are classicaly separeted into three contributions (Bouzaiane et al (2004)):

$$
\begin{aligned}
& \varphi_{i j}=\varphi_{i j}^{1}+\varphi_{i j}^{2}+\varphi_{i j}^{3} \\
& \varphi_{i \rho}=\varphi_{i \rho}^{1}+\varphi_{i \rho}^{2}+\varphi_{i \rho}^{3}
\end{aligned}
$$

Here, terms noted 1 are terms of return to the isotropy, they characterize the non linear mechanism of interaction between turbulent fluctuations. The terms 2 represent the interaction between mean and turbulent flows, they characterize the linear terms .Finally, the terms 3 are terms due to buoyancy effects (Bouzaiane et al. (2004)). During the two past decades several models have been presented by authors. Perhaps the Speziale Sarkar and Gatski model for pressurestrain correlation is among the most interesting one. For its great success during the last decade, the SSG model (Speziale et al. (1990)) is retained in this work. We precise here that to our knowledge this model has not been extented to scalar effects present in our stratified turbulent flow. A coupling between the SSG model retained for kinematic field and three of the most known models for scalars field is proposed and makes the motivation of this part of our work.

\subsection{The Speziale Sarkar and Gatski (SSG) model}

This model concerns only kinematic turbulence, Speziale et al. (1990) separated the part of the return to the isotropy from the linear part. This model is written in the following form:

$$
\begin{aligned}
& \varphi_{i j}=\varphi_{i j}^{1}+\varphi_{i j}^{2} \\
& \varphi_{i j}{ }^{1}=-C_{1} b_{i j}+3\left(C_{1}-2\right)\left(b_{i j}^{2}+I I_{b} \frac{\delta_{i j}}{3}\right) \\
& I_{b}=b_{k l} b_{l k}, C_{1}=3.4 \\
& \phi_{i j}{ }^{2}=C_{2} b_{m n} S_{m n} b_{i j}+\frac{1}{2}\left(C_{3}-\sqrt{b_{m n} b_{m n}} C_{33}\right) S_{i j}+ \\
& \frac{1}{2} C_{4}\left(b_{i k} S_{k j}+b_{j k} S_{k i}-\frac{2}{3} b_{m n} S_{m n} \delta_{i j}\right)+\frac{1}{2} C_{5}\left(b_{i k} w_{j k}+b_{j k} w_{i k}\right)
\end{aligned}
$$




$$
\begin{aligned}
& \text { Where } C_{2}=1.8 \quad C_{3}=0.8 \quad C_{4}=1.25 \\
& C_{5}=0.4 \quad C_{33}=1.3
\end{aligned}
$$

Speziale et al. (1990) supplemented their models of the pressure-strain correlation by the following model of the equation of viscous dissipation $\varepsilon$ :

$$
\frac{d \varepsilon}{d t}=-2 C_{\varepsilon 1} \frac{\varepsilon}{k} b_{m l} U_{l, m} S-C_{\varepsilon 2} \frac{\varepsilon^{2}}{k}+C_{\varepsilon 3}
$$

Where $C_{\varepsilon 1}=1.45 \quad C_{\varepsilon 2}=1.9$

This model as mentioned is not extended to scalars effects, it will be coupled respectively to the classic Launder, Reece and Rodi (LRR) model (Launder et al. (1975), Cadiou (1996b)), the Craft and Launder (CL) model (Craft el al. (1989), Launder et al. (1996) and Launder (1999)) and the Shih and Lumley (SL) model (Shih et al. (1989) and Shih (1996)). This coupling will be noted respectively model 1 , model 2 and model 3.

We note also that the classic popular model of Zeman and Lumley (1976) is the only model retained for the third contribution of pressure-strain correlation and pressure-scalar gradient correlation. These models are written as follows:

$$
\begin{aligned}
& \phi_{i j}^{3}=-C_{3}\left(\beta_{j} \overline{u_{i} \rho}+\beta_{i} \overline{u_{j} \rho}-\frac{2}{3} \beta_{l} \overline{u_{l} \rho} \delta_{i j}\right) \\
& C_{3}=0.5 \\
& \phi_{i \rho}^{3}=-C_{3 \rho} \beta_{i} \overline{\rho^{2}} \\
& C_{3 \rho}=0.5
\end{aligned}
$$

Where $\beta_{i}$ is the term of gravity $\beta_{i}=\frac{g_{i}}{\rho_{0}}$.

\subsection{Non-dimensional Equation}

With the goal of getting non-dimensional equations, a closed system of non-dimensional parameters can be obtained by casting basic Eqs.(4), (5) and (6) in non-dimensional form and by introducing the nondimensional time $\tau=S t$, the components $b_{i j}=\frac{\overline{u_{i} u_{j}}}{2 k}-\frac{\delta_{i j}}{3}$ of the anisotropic tensor $\mathrm{b}$ (Schiestel (1997)) and the non-dimensional shear number $\frac{\varepsilon}{K S}$ are classically (Jacobitz et al. (1999b)) retained for the kinematic field. The component of the non-dimensional turbulent scalar flux (Petterson et al. (2000)) $F_{i}=\frac{g}{\rho_{0}} \frac{\overline{u_{i} \rho}}{k S}$ and the ratio $\eta=\frac{K \rho}{K}$ of potential energy to kinetic energy substitute respectively the turbulent scalar flux and the variance of scalar to get a closed form of differential equation for the scalar field.

$$
\frac{d b_{11}}{d \tau}=-2 b_{12}+\frac{\phi_{11}}{2 k S}-\frac{\varepsilon}{3 k S}+\left(b_{11}+\frac{1}{3}\right)\left(2 b_{12}+F_{3}+\frac{\varepsilon}{k S}\right)
$$

$$
\begin{aligned}
& \frac{d b_{22}}{d \tau}=\frac{\varphi_{22}}{2 k S}-\frac{\varepsilon}{3 k S}+\left(b_{22}+\frac{1}{3}\right)\left(2 b_{12}+F_{3}+\frac{\varepsilon}{k S}\right) \\
& \frac{d b_{12}}{d \tau}=-\left(b_{22}+\frac{1}{3}\right)+\frac{\phi_{12}}{2 k S}+2 b_{12}^{2}+F_{3} b_{12}+\left(\frac{\varepsilon}{k S}\right) b_{12} \\
& \frac{d b_{13}}{d \tau}=-b_{23}-\frac{1}{2} F_{1}+\frac{\phi_{13}}{2 k S}+2 b_{12} b_{13}+\left(F_{3}+\frac{\varepsilon}{k S}\right) b_{13} \\
& \frac{d b_{23}}{d \tau}=-\frac{1}{2} F_{2}+\frac{\phi_{23}}{2 k S}+2 b_{12} b_{23}+\left(F_{3}+\frac{\varepsilon}{k S}\right) b_{23} \\
& \frac{d F_{1}}{d \tau}=-S F_{2}-2 R_{i} b_{13}+\frac{\phi_{1 \rho}}{S^{2}}\left(\frac{g}{\rho_{0} k}\right)+F_{1}\left(2 b_{12}+F_{3}+\frac{\varepsilon}{k S}\right) \\
& \frac{d F_{2}}{d \tau}=-2 R_{i} b_{23}+\frac{\phi_{2 \rho}}{S^{2}}\left(\frac{g}{\rho_{0} k}\right)+F_{2}\left(2 b_{12}+F_{3}+\frac{\varepsilon}{k S}\right) \\
& \frac{d F_{3}}{d \tau}=-2 R_{i}\left(b_{33}+\eta\right)+\frac{\phi_{3 \rho}}{S^{2}}\left(\frac{g}{\rho_{0} k}\right)+F_{3}\left(2 b_{12}+F_{3}+\frac{\varepsilon}{k S}\right) \\
& \frac{d \eta}{d \tau}=-F_{3}+\eta\left(2 b_{12}+F_{3}-\frac{\varepsilon}{k S}\right) \\
& \frac{d}{d \tau}\left(\frac{\varepsilon}{k S}\right)=-2 C_{\varepsilon 1}\left(\frac{\varepsilon}{k S}\right) b_{13}+\left(1-C_{\varepsilon 2}\right)\left(\frac{\varepsilon}{k S}\right)^{2} \\
& -C_{\varepsilon 1}\left(1-C_{\varepsilon 3}\right)\left(\frac{\varepsilon}{k S}\right) F_{3}+\left(\frac{\varepsilon}{k S}\right)\left(2 b_{12}+F_{3}\right)
\end{aligned}
$$

It is essential to note here that the expressions of models of $\phi_{i j}$ and $\phi_{i \rho}$ let us to write the quantities $\frac{\phi_{i j}}{2 k S}$ and $\frac{\phi_{i \rho}}{\rho_{0} k S}$ in terms of non-dimensional parameters $\mathrm{b}_{\mathrm{ij}}, \mathrm{F}_{\mathrm{i}}, \frac{\varepsilon}{k S}$ and $\eta$.

In this step of our work, numerical integration of the above differential equation is started. Discussions of obtained result will make the object of the following sub-sections.

\section{NUMERICAL INTEGRATION AND RESULTS}

A fourth order Runge-Kutta method is used for integrating the non-dimensional system of ten nonlinear differential equations submitted to the initial conditions of the results of the Direct Numerical Simulation of Jacobitz (2002) and Jacobitz et al. (1998). A comparison between obtained results and results of the Direct Numerical Simulation (DNS) of Jacobitz (1998) forms a part of this section.

\subsection{Influence of the Gradient Richardson Number}

Numerical integration is conducted to long time evolution $\tau=$ St. Evolution of the principal component of anisotropy $b_{12}$ as a function of non dimensional time $\mathrm{St}$ is presented in Fig. 2. A general tendency to asymptotic equilibrium states has been observed for $b_{12}$ as long time evolution $\tau=$ St. 
In Table 1 , asymptotic equilibrium values of $b_{12}$ for differents values of Richardson number $\mathrm{Ri}=0.2$, $\mathrm{Ri}=0.4, \mathrm{Ri}=0.6$ and $\mathrm{Ri}=1.0$ and $\varepsilon / \mathrm{KS}=0.5$ reached by models are presented:

Table 1 Asymptotic equilibrium values of $\left(b_{12}\right)_{\infty}$ for $\boldsymbol{\varepsilon} / \mathrm{KS}=0.5$

\begin{tabular}{|lcccc|}
\hline \multicolumn{5}{|c|}{$\left(\mathrm{b}_{12}\right)_{\infty}$} \\
\hline Models & $\begin{array}{c}\text { Model } \\
1\end{array}$ & $\begin{array}{c}\text { Model } \\
2\end{array}$ & $\begin{array}{c}\text { Model } \\
3\end{array}$ & $\begin{array}{c}\text { DNS } \\
\text { of J }\end{array}$ \\
\hline & & & & -0.13 \\
$\mathrm{Ri}=0.2$ & -0.124 & -0.134 & - & -0.11 \\
$\mathrm{Ri}=0.4$ & -0.118 & -0.127 & 0.0783 & - \\
$\mathrm{Ri}=0.6$ & -0.115 & -0.125 & - & -0.115 \\
$\mathrm{Ri}=1$ & -0.110 & -0.119 & 0.0739 & -0.1 \\
& & & - & \\
& & & 0.0712 & \\
& & & 0.0701 & \\
\hline
\end{tabular}

The three models confirm the existence of an asymptotic equilibrium states for the component $\mathrm{b}_{12}$, for different values of Richardson number $\mathrm{Ri}$ and $\varepsilon / \mathrm{KS}=0.5$. However a principal result is observed and showed the positive contribution of model SSG on the prediction of equilibrium state of the field scalar:

In a previous work (Melki et al. (2010)) the LRR model retained on its individual for both kinematic and scalar fields has not predicted an asymptotic equilibrium states for any dimensionless parameters. Here the coupling between the SSG model for kinematic field and LRR model for scalar field (SSG-LRR, model 1) indicates existence of asymptotic equilibrium states for $b_{12}$ for all retained values of Richardson number Ri.

This result constitutes the first positive contribution of the SSG model when it is coupled with LRR model. In Fig.2, also we see that the coupling between the SSG and LRR (model 1) indicates the best agreement with the values of DNS of Jacobitz, compared with values predicted by model 2 ( SSG$\mathrm{CL}$ ) in one hand and model 3 (SSG-SL) in the other hand for non dimensional time $\tau=\mathrm{St}$ greater than 25 . In the first period corresponding to St less than 25 , no agreement between predictions of models and values of DNS of Jacobitz has been observed.

An excellent agreement between the prediction of the model 1 and the values of DNS of Jacobitz is observed. A qualitative agreement between the predictions of two other models 2 and 3 on one hand and the results of DNS of Jacobitz on the other hand is also observed. The model 2 shows a good agreement with these values only for dimensionless time $\tau$ greater than 30 ( $\tau \geq 30$ ).

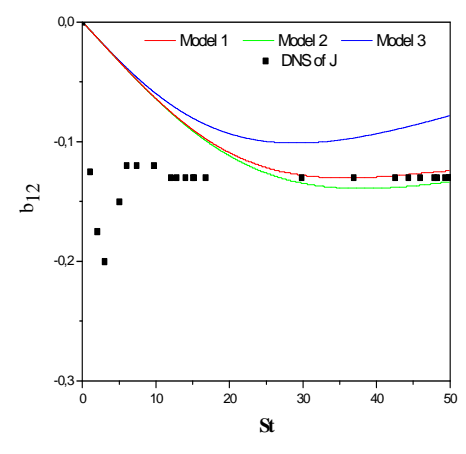

Fig. 2. Time evolution of the component $b_{12}$ for $\mathrm{Ri}=0.2$ and $\boldsymbol{\varepsilon} / \mathrm{KS}=0.5$

Thereafter, we present the influence of the Richardson number $\mathrm{Ri}$ in one hand and the influence of the initial non-dimensional number $\varepsilon / \mathrm{KS}$ in the other hand respectively on the principal component of anisotropy $b_{12}$, on the rate of dimensionless shear number $\varepsilon / \mathrm{KS}$, on the turbulent kinetic energy $\mathrm{K}$ and the potential energy $\mathrm{K}_{\rho}$ for the three retained models.
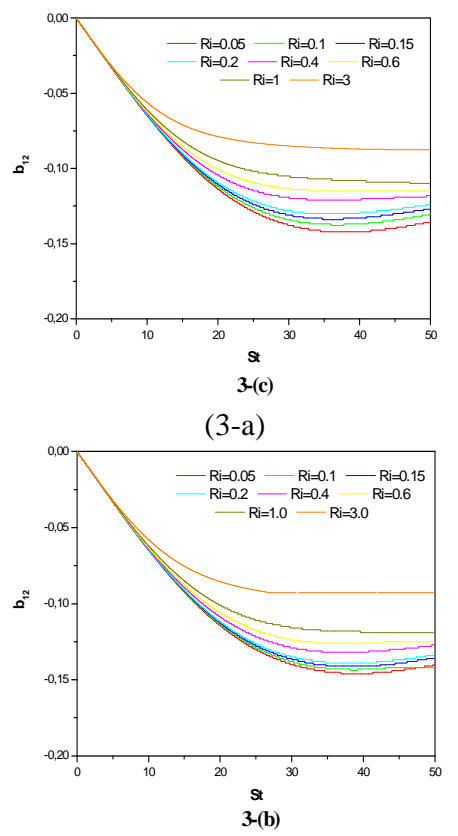

(3-b)

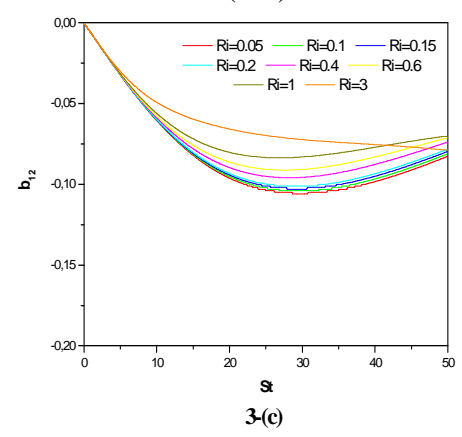

(3-c)

Fig. 3. Time evolution of the component $b_{12}$ for different values of $\mathbf{R i}$.

3-(a) model 1, 3-(b) model 2; 3-(c) model 3 
Figures 3-(a), 3-(b) and 3-(c) show the evolution of the principal component of anisotropy $b_{12}$ as a function of the non-dimensional time $\mathrm{St}=\tau$, obtained by the model 1 , model 2 and model 3 respectively and for different values of the gradient Richardson number. Three models confirm the existence of an asymptotic equilibrium states for the component $b_{12}$. Three models indicate also that $\left(b_{12}\right)_{\text {oo }}$ grows with $\mathrm{Ri}$ growing from weak stratification $(\mathrm{Ri}=0.05)$ to strong stratification $(\mathrm{Ri}=3.0)$. This result is not in contradiction with our previous results (Bouzaiane et al. (2004)). We note also that the asymptotic equilibrium state for the model 1 and the model 2 are reached very quickly compared to the prediction of the model 3 which predict an equilibrium state from $\mathrm{St}=\tau=48$.

\subsection{Influence of the Initial Value of $\varepsilon / K S$ on Equilibrium State of $b_{12}$}

In Table 2, the equilibrium values of $b_{12}$ predicted by several retained models and for results of DNS of Jacobitz et al. (1999b) are presented for values $1 / 2,1 / 6,1 / 10$ and $1 / 14$ of $\varepsilon / \mathrm{KS}$, and for $\mathrm{Ri}=0.15$. The principal result presented in Table 2 is surprising, since three models show a growth of the absolute value of $\left(b_{12}\right)_{o o}$ for a decrease of the initial value of $(\varepsilon / \mathrm{KS})$. This result is in a clear contradiction of the result of DNS of Jacobitz.

Table 2 Asymptotic equilibrium values of $\left(b_{12}\right)_{\infty}$ for $\mathbf{R i}=\mathbf{0 . 1 5}$

\begin{tabular}{|lcccc|}
\hline \multicolumn{5}{c|}{$\left(\mathrm{b}_{12}\right)_{\infty}$} \\
\hline Models & $\begin{array}{c}\text { Model } \\
1\end{array}$ & $\begin{array}{c}\text { Model } \\
2\end{array}$ & $\begin{array}{c}\text { Model } \\
3\end{array}$ & $\begin{array}{c}\text { DNS } \\
\text { of J }\end{array}$ \\
\hline & & & & \\
$\varepsilon / \mathrm{KS}=1 / 2$ & -0.127 & -0.136 & - & -0.1 \\
$\varepsilon / \mathrm{KS}=1 / 6$ & -0.134 & -0.141 & 0.0797 & - \\
$\varepsilon / \mathrm{KS}=1 / 10$ & -0.136 & -0.145 & -0.111 & 0.075 \\
$\varepsilon / \mathrm{KS}=1 / 14$ & -0.137 & -0.147 & -0.128 & -0.05 \\
& & & -0.138 & -0.04 \\
\hline
\end{tabular}

In Figs.4-(a), 4-(b) and 4-(c) are presented evolutions predicted by the three retained models, model 1, model 2 and model 3. Asymtotic equilibrium states are reached for $\tau=S$ t greater than 20. The asymptotic value of the principal component of anisotropy $b_{12}$ decreases strongly when the initial value of the shear number is increased. An excellent agreement for $\varepsilon / \mathrm{KS}=1 / 2$ for the three models and the values of DNS of Jacobitz et al. (1999b) is observed. Model 2 shows a good agreement with these values only for a dimensionless time $\tau(\tau \geq 20$ ).

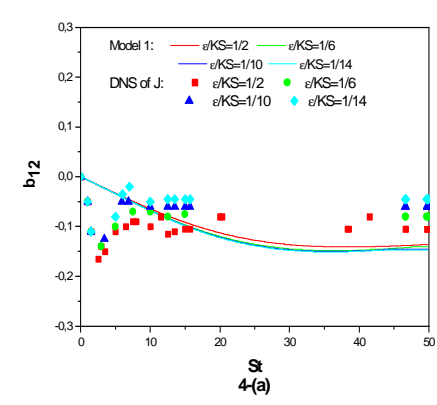

(4)-a

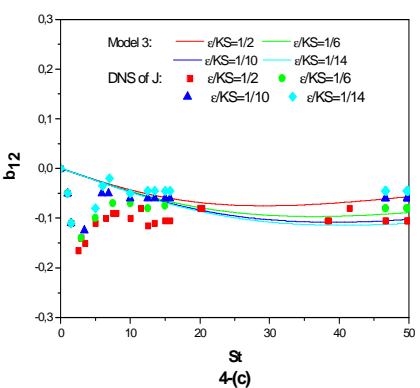

(4)-b

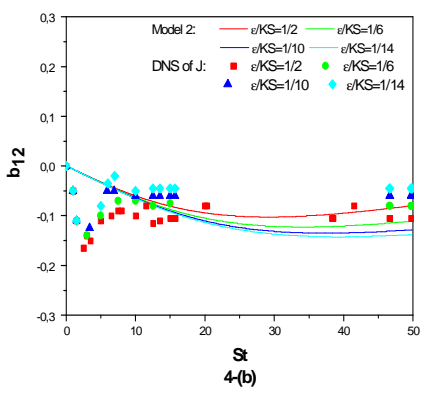

(4)-c

Fig. 4. Time evolution of the component $b_{12}$ for different values of $\varepsilon / K S$ and $R i=0.15$.

4-(a) model 1, 4-(b) model 2, 4-(c) model 3

\subsection{Growth Rate of the Turbulent Kinetic Energy}

An interesting other non dimensional parameter is generally introduced to characterize the time evolution of the turbulent kinetic energy. The growth rate of turbulent kinetic energy is mathematically defined as:

$\gamma=\frac{1}{S K} \frac{d K}{d t}=\frac{P}{S K}-\frac{B}{S K}-\frac{\varepsilon}{S K}$

It is clear that the growth rate $\gamma$ depends on the normalized production term $\frac{P}{S K}$, the normalized 
buoyancy flux $\frac{B}{S K}$, and the normalized dissipation rate $\frac{\varepsilon}{S K}$.

Figure 5, shows the evolution of the growth rate $\gamma$ of the turbulent kinetic energy. An asymptotically constant value of $\gamma$ is reached for non dimensional time greater than 20 (St >20). Model 1 shows an over estimation of asymptotic equilibrium states of $\gamma$, whereas, the two other models show a very good estimation of values of DNS of Jacobitz for $\tau$ greater than 30 .

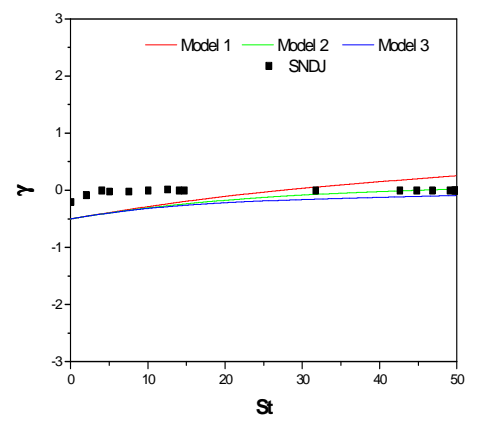

Fig. 5. Evolution of the growth rate $\gamma$ for $R i=0.15$ and for $\varepsilon / K S=0.2$

In Figs.6-(a), 6-(b) and 6-(c), we show the evolution of the non-dimensional shear number $\varepsilon / \mathrm{KS}$ for $\mathrm{Ri}=0.15$ and for differents initial values of $\varepsilon / \mathrm{KS}$, according to the three retained second-order models model 1 , model 2 and model 3, respectively. We notice that predictions of the three models tend towards to equilibrium states and that when the non-dimensional number $\varepsilon / \mathrm{KS}$ increases from $\varepsilon / \mathrm{KS}=1 / 20$ to $\varepsilon / \mathrm{KS}=1 / 2$, the ratio of nondimensional shear $\varepsilon / \mathrm{KS}$ increases too. The asymptotic values of the non dimensional shear number $\varepsilon / K S$ decrease as the initial value of the shear number is increased. The asymptotic values of the normalized dissipation rate $\varepsilon / \mathrm{KS}$ decrease as the initial value of the shear number is increased.

The dependence of the non dimensional shear number $\varepsilon / \mathrm{KS}$ for non dimensional time $\mathrm{St}$ is shown in Fig.7 for the three retained second-order models and compared with values of DNS of Jacobitz et al. (1999b) for $\mathrm{Ri}=0.15$ and $\varepsilon / \mathrm{KS}=1 / 6$.

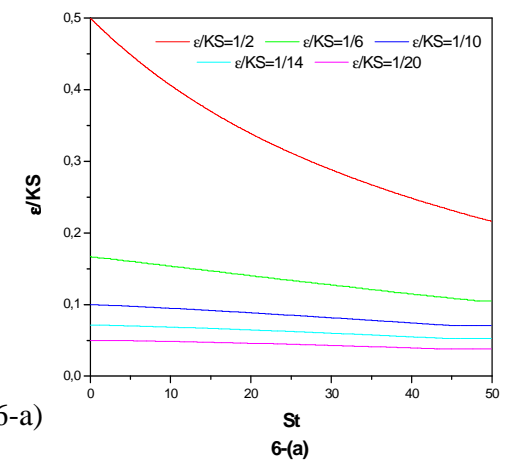

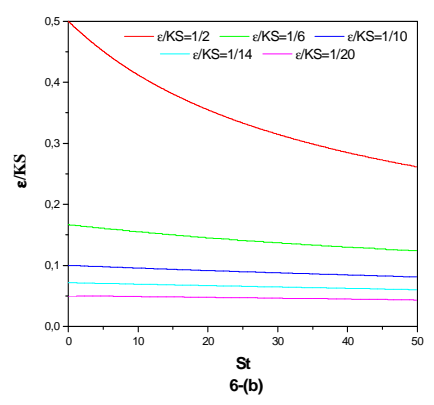

(6-b)

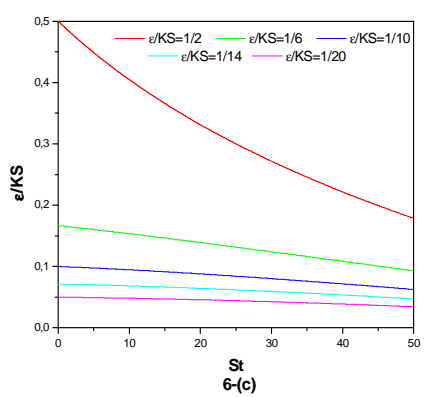

(6-c)

Fig. 6. Evolution of the normalised dissipation rate $\varepsilon / K S$ for $R i=0.15$, 6-(a) model 1, 6-(b) model 2, 6-(c) model 3

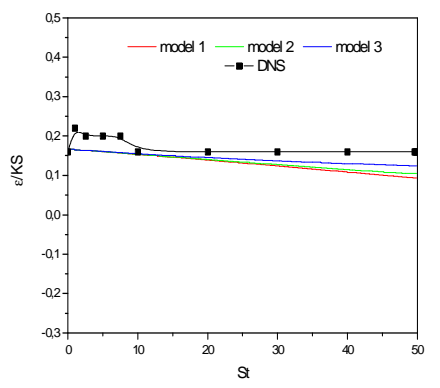

Fig. 7. Evolution of the normalized dissipation rate $\varepsilon / \mathrm{KS}$ for $\mathrm{Ri}=0.15$ and $\varepsilon / \mathrm{KS}=1 / 6$

The asymptotic equilibrium behavior of nondimensional parameters $\mathrm{b}_{12}, \mathrm{~B} / \varepsilon$ and $\varepsilon / \mathrm{KS}$ allows us to write the time-evolution equations of the turbulent kinetic energy $K$ and $\varepsilon$ in the following form:

$\frac{d K}{d \tau}=\left(-2 b_{12}-F_{3}-\frac{\varepsilon}{S K}\right) K$

When $\tau \rightarrow \infty$, the non-dimensional parameters approach constant values and the above equation becomes a first-order differential equation with constant coefficient and take the following form:

$$
\frac{d K}{d \tau}=\alpha_{K} K
$$


Where: $\alpha_{K}=2 b_{12}-F_{3}-\frac{\varepsilon}{S K}$

Finally, as $\tau \rightarrow \infty$ we have $K=K(0) \exp \left(\alpha_{K} \tau\right)$.

\subsection{Evolutions of Ratio $\mathrm{K} / \mathrm{E}$ and $\mathrm{K}_{\mathrm{\rho}} / \mathrm{E}$}

The influence of the Richardson number Ri on the dimensionless ratios $\mathrm{K} / \mathrm{E}$ and $\mathrm{K}_{\rho} / \mathrm{E}$ is also analysed. Figure 8, shows the influence of gradient Richardson number on $\mathrm{K} / \mathrm{E}$ and $\mathrm{K}_{\mathrm{\rho}} / \mathrm{E}$, we see here, that at high stratification corresponding to $\mathrm{Ri}=1.0$ and $\mathrm{Ri}=2.0$, the ratio of potential energy to total energy $K_{\rho} / E$ and kinetic energy total $K / E$ have a tendency to reach the numerical value 0.5 for $\tau=\mathrm{St}>40$.

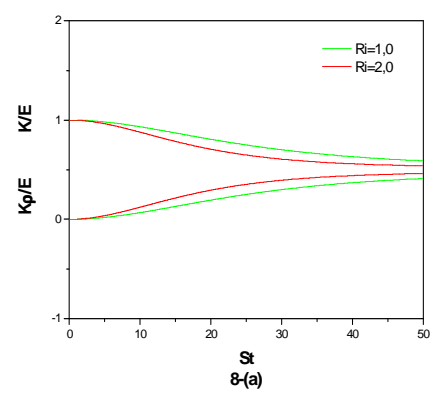

$(8-\mathrm{a})$

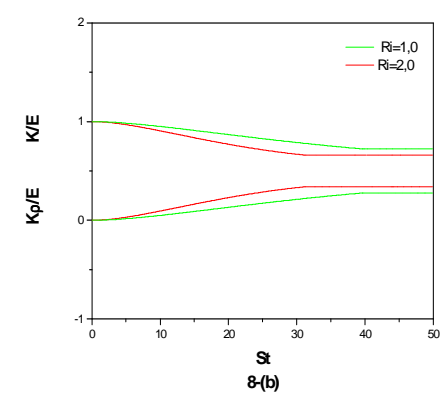

(8-b)

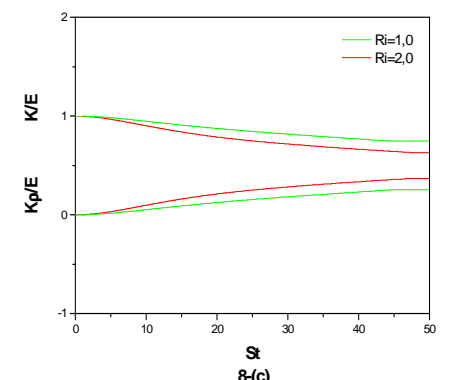

(8c)

Fig. 8.Time evolution of the ratios $\mathrm{K} / \mathrm{E}$ and $K_{\rho} / E, R i=2.0$ and $R i=1.0$

8-(a) model 1, 8-(b) model 2, 8-(c) model 3

We add also that at high stratification (see Fig. 8 -(a)) the asymptotic equilibrium values of $\mathrm{K} / \mathrm{E}$ and $\mathrm{K}_{\mathrm{\rho}} / \mathrm{E}$ are slightly different from the mean value 0.5 . The model 1 shows an equal partition of the total energy $\left(E=K+K_{\rho}\right)$ between the turbulent kinetic energy and potential energy. These Figs.8-(a), 8-(b) and $8-(\mathrm{c})$, confirm the existence of equilibrium states for three models summarized in Tab.3. We also notice that when the Richardson number $\mathrm{Ri}$ increases from 1.0 to 2.0, the ratio of kinetic energy decreases whereas the ratio of potential energy increases progressively. This prediction is coherent, since when Ri grows from 1.0 to 2.0, the effects of scalar dominates the effect of shear.

Table 3. Asymptotic equilibrium values of (K/E)
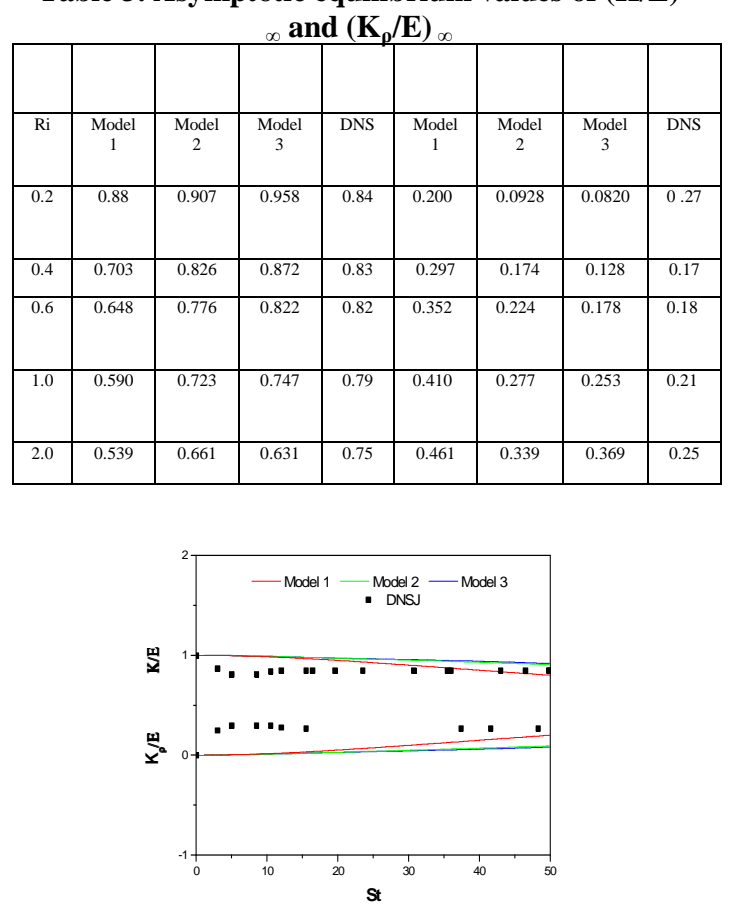

Fig. 9.Time evolution of the ratios $\mathrm{K} / \mathrm{E}$ and $\mathrm{K}$ $\rho \mathrm{E}, \mathrm{Ri}=0.2$

In Fig.9, the time evolution of the dimensionless ratios $\mathrm{K} / \mathrm{E}$ and $\mathrm{K}_{\rho} / \mathrm{E}$ are presented. Here $\mathrm{E}$ is the total energy. For the ratio $\mathrm{K} / \mathrm{E}$, it is clear that model 1 ensures the best agreement with the results of the DNS of Jacobitz Jacobitz (1998) for nondimensional time greater than $40(\tau \geq 40)$ and a qualitative agreement between the predictions of two other models (model 2 and model 3 ) on the one hand and the results of DNS of Jacobitz (1998) on the other hand is also observed. For values of dimensionless time greater than 40 , model 2 , model 3 and model 1 show respectively an underestimation of $15 \%, 30 \%$ and $35 \%$ of the values of DNS of Jacobitz. For the ratio $\mathrm{K}_{\rho} / \mathrm{E}$ no good agreement has been observed between values predicted by the retained second-order models and the values of DNS of Jacobitz (1998).

\section{CONCLUSION}

In this study we have investigated a stably stratified turbulence submitted to an horizontal shear. Two approaches have been retained. A first one is analytic and is based on a linear solution when non linear effects of pressure and viscosity are neglected in time evolution equation. A Laplace Transform has been used for integrating ten linear differential equations. Obtained solutions have confirmed at 
long time evolution the existence of asymptotic equilibrium behavious for dimensionless kinematics and scalars parameters.

The second approach is a numerical one and is based on a second-order modeling of pressure-strain and pressure-scalar gradient correlation, besides, time evolution equation of dissipation rates. The SSG model has been retained for pressure-strain correlation and dissipation equation, whereas three of the most known models are retained for pressure scalar gradient correlations. Equations are castled in non dimensional form when non dimensional parameters are introduced for both kinematic and scalar fields. A fourth order Runge Kutta method has been used to integrate three non linear differential equations submitted to the initial condition of the results of DNS of Jacobitz et al. (1999a).

The principal results obtained in this work are:

- Asymptotic equilibrium behavior of dimensionless kinematic and scalar parameters have been confirmed by linear solutions obtained when non linear effects of pressure and viscosity have been neglected according to the results of DNS of Holt.

-Analytic equilibrium solutions are functions only of the initial conditions and the gradient Richardson number Ri.

- The existence of asymptotic equilibrium states of dimensionless kinematic and scalar parameters for the three retained second order models are generally observed.

-The Coupling between the SSG model for the kinematic field and the LRR model for the scalars has been of a big contribution in predicting asymptotic equilibrium states. In fact the LRR model retained in its individual for both kinematic and scalar fields does not predict a such behavior (Bouzaiane et al. (2003c)).

- For the influence of initial value of the non dimensional number $\varepsilon / \mathrm{KS}$ on equilibrium state of $\mathrm{b}_{12}$, no agreement between results of the three retained second order models and results of DNS of $\mathrm{J}$ have been observed.

- Model 1 (SSG-LRR) shows the better agreement with the results of DNS of Jacobitz for the predictions of the component $b_{12}$ of the tensor of anisotropy of Reynolds and the ratio of kinetic energy to total energy K/E.

- The Speziale, Sarkar and Gatski (SSG) model has been of a considerable contribution in the modeling of turbulence by improving predictions of different models.

We think that the present work can be extended according to several directions. A correction to models in a similar manner as a previous work of Hechmi et al. (2012) to improve effects of the non dimensional number $\varepsilon / \mathrm{KS}$ in turbulent parameters can make a coherent extension to this work. The study of the coupling effects of stratification and rotations in turbulent parameters, by the two different methods, seems also an important direction of investigations.

It is important here to note that stratification turbulence is present in several engineering and environmental application. Examples are flow over topography, river in flow into the ocean or effluent discharge by power plants (Jacobitz (2002)).

\section{Appendix A: Coefficients of analytical solutions}

$\beta=\frac{g}{\rho_{0}} \quad \alpha=1 \quad \gamma=0$

$C_{11}=y_{1}(0)$

$A_{11}=-2 y_{4}(0)$

$B_{11}=2 y_{2}(0)$

$D_{11}=0$

$E_{11}=0$

$F_{11}=0$

$C_{33}=y_{3}(0)-\left(\frac{y_{3}(0)}{2}+\frac{\beta^{2} y_{10}(0)}{2 S^{2} R_{i}}\right)$

$F_{33}=\frac{\beta y_{9}(0)}{\sqrt{R_{i} S}}$

$K_{33}=\left(\frac{y_{3}(0)}{2}+\frac{\beta^{2} y_{10}(0)}{2 S^{2} R_{i}}\right)$

$C_{12}=y_{4}(0)$

$A_{12}=y_{2}(0)$

$B_{12}=0$

$C_{13}=y_{5}(0)-\frac{\beta}{S} \frac{y_{8}(0)}{R_{i}}$

$A_{13}=\frac{\beta}{S} \frac{y_{8}(0)}{R_{i}}$

$B_{13}=y_{5}(0)+\frac{\beta}{S} \frac{y_{8}(0)}{R_{i}}$

$D_{13}=\left\{-\frac{\beta}{S} \frac{y_{7}(0)}{\sqrt{R_{i}}}-2 \frac{y_{6}(0)}{\sqrt{R_{i}}}-\frac{3}{2 \sqrt{R_{i}} R_{i}}\left[\frac{\beta}{S} y_{8}(0)-R y_{6}(0)\right]\right\}$

$E_{13}=\frac{\beta}{S} \frac{y_{8}(0)}{2 \sqrt{R_{i}}} \frac{1}{2}\left[\frac{\beta}{S} \frac{y_{8}(0)}{R_{i}}-y_{6}(0)\right]$

$F_{13}=\frac{\beta}{S} \frac{y_{8}(0)}{2 \sqrt{R_{i}}}$

$K_{13}=0$

$C_{23}=y_{6}(0)$ 


$$
\begin{aligned}
& A_{23}=-\frac{\beta}{S} y_{8}(0) \\
& B_{23}=y_{6}(0) \sqrt{R_{i}} \\
& C_{1 \rho}=-\frac{y_{8}(0)}{R_{i}} \\
& A_{1 \rho}=\left\{y_{7}(0)+\frac{S_{\rho}}{S} \frac{y_{6}(0)}{R_{i}}\right\} \\
& B_{1 \rho}=-\frac{S_{\rho}}{S} \frac{y_{5}(0)}{\sqrt{R_{i}}}+\frac{1}{2 \sqrt{R_{i}}} \frac{S_{\rho}}{S} y_{6}(0) \tau-\frac{1}{2} \frac{y_{8}(0)}{\sqrt{R_{i}}} \\
& D_{1 \rho}=\left\{-\frac{1}{2 \sqrt{R_{i}}}\left(\frac{S_{\rho}}{S} \frac{y_{6}(0)}{R_{i}}-\frac{\left.y_{8}(0)\right)}{R_{i}}\right)\right\} \\
& E_{1 \rho}=0 \\
& F_{1 \rho}=0 \\
& A_{2 \rho}=y_{8}(0) \\
& B_{2 \rho}=-\frac{S_{\rho}}{S} \frac{y_{6}(0)}{\sqrt{R_{i}}} \\
& A_{3 \rho}=y_{9}(0) \\
& B_{3 \rho}=-\left[\frac{S_{\rho}}{S} \frac{y_{3}(0)}{2 \sqrt{R_{i}}}+\frac{\beta}{S} \frac{y_{10}(0)}{2 \sqrt{R_{i}}}\right] \\
& C_{\rho}=\frac{y_{10}(0)}{2}-\left(\frac{S_{\rho}}{S}\right)^{2} \frac{y_{3}(0)}{2 R_{i}} \\
& H_{q}=-\frac{\beta}{\sqrt{R_{i}}} \frac{y_{9}(0)}{S} \\
& A_{\rho}=\left[\left(\frac{S_{\rho}}{S}\right)^{2} \frac{y_{3}(0)}{2 R_{i}}+\frac{y_{10}(0)}{2}\right] \\
& A_{q}=0 \\
& B_{q}=2 y_{y_{2}}(0)+y_{2}(0)+\frac{y_{9}(0)}{\sqrt{R_{i}}} \\
& D_{q}=0 \\
& E_{q}=0 \\
& F_{q}=\left[\frac{y_{3}(0)}{2}+\frac{\beta^{2} y_{10}(0)}{2} S^{2}\right. \\
& B_{4}(0)
\end{aligned}
$$

\section{REFERENCES}

Ben Abdallah, H., Bouzaiane, M., Lili, T. (2005), A study of equilibrium states of homogeneous turbulence with a stable stratification and a non vertical shear, Phys.Chem.News.23, pp. 34-46.

Bouzaiane, M., Ben Abdallah, H. and Lili, T. (2004), A second order modeling of a stably stratified sheared turbulence submitted to a non vertical shear, Journal of turbulence, 5, 118.

Bouzaiane, M., Ben Abdallah, H., Lili, T. (2003a), Solutions analytiques en turbulence stratifiée soumise à un cisaillement non vertical, SFT2003, Congres Français de thermique.

Bouzaiane, M., Ben Abdallah, H., Lili, T. (2003b), A study of a stably stratified turbulence submitted to a non-vertical shear, Mechanics and materials conference Arizona State University.

Bouzaiane, M., Ben Abdallah, H., Lili, T. (2003c), A study of the asymptotic behaviour in a stably stratified sheared turbulence, Journal of turbulence, 4, 002.

Cadiou, A. (1996a), A contribution to the study of second order turbulence closure models Ph.D.thesis, Central school of Nantes France.

Cadiou, A. (1996b), Contribution à l'étude de modèle de turbulence au second ordre, thèse de doctorat, école centrale de Nantes.

Caldwell, D. R. (1987), Small-scale physics of the ocean, Rev.Geophys.25, 183-192.

Chebbi, B. , Bouzaiane, M. (2012), On the effects of rotation on the passive scalar and kinematic fields of homogeneous sheared turbulence, Journal of Applied Fluid Mechanics, 5(2), 5565.

Craft, T.J. and Launder, B.E. (1989), A model for the pressure scalar gradient correlation and its application to homogeneous and inhomogeneous shear flow, $7^{\text {th }}$ turbulent shear flow, Stanford University press.12, 1-6.

Jacobitz, F. (2002), Acomparaison of the turbulence evolution in a stratified fluid with vertical or horizontal shear, Journal of Turbulence, 3, 118.

Jacobitz, F. and Sarkar, S. (1999a), A direct numerical study of transport and anisotropy in a stably stratified turbulent flow with uniform horizontal shear, Flow Turbulence and combustion 63, 343-360.

Jacobitz, F., Sarkar, S. (1999b), On the shear number effect in stratified shear flow, Theoretical and computational fluid dynamics.13, 171-188. 
Jacobitz, F., Sarkar, S. (1998), The effect of non vertical shear on turbulence in a stably stratified medium, Phys.Fluid.10, 1158-1168.

Jacobitz, F., Sarkar, S., Van Atta, C. (1997), Direct numerical simulations of the turbulence evolution in a uniformly sheared and stably stratified flow, J.Fluid Mech.342, 231-261.

Hechmi, K. and Lili. , T. (2012), An Extension of the SSG Model on Compressible Turbulent Flow, Journal of Applied Fluid Mechanics, 5, 101-111.

Holt, S., Ferziger, J. (1992), A numerical study of the evolution and structure of homogeneous stably stratified sheared turbulence, J.Fluid Mech.237, 499-539.

Gerz, T., Shumann, U., Elghobachi, S. (1989), Direct numerical simulation of stratified homogeneous turbulent shear flow, J.Fluid Mech.200, 563-594.

Khaleghi, K., M.Pasandideh. Fard, M. Malek. Jafarian and Y.M Chung (2010).Assessment of Common Turbulence Models under Conditions of Temporal Acceleration in a Pipe. Journal of Applied Fluid Mechanics 3(1), 25-33.

Komori, S., Ueda, H., Ogino, F. and Mizushina, T. (1983), Turbulence structure in stably stratified open-channel flow, J.Fluid Mech.130, 13.

Launder, B.E. (1999), Principales and performance of TCL based second moment closures commemorative conference HA MINH THIEU Toulouse, 1-19.

Launder, B.E., Reece, G., Rodi, W. (1975), Progress in the developmenty of a Reynolds stress closure, J.Fluid.Mech.68, 537-576.

Launder, B.E., Reece, G., Rodi, W. (1996), Advanced turbulence models for industrial application, in turbulence transition modeling edited by M.Hallback and D.S.Hennigson and A.V. Johansson and P.H. Alfredsson, Dordrecht.

Melki, L., Lili, T., Bouzaiane, M. (2010), A second order modelling of a stably stratified turbulence using the SSG model for the kinematic field, International Review of Physics.4.
Petterson Reif, B. A., Ooi, A. and Durbin, P. A. (2000), On stably stratified shear flows subjected to rotation, Center for Turbulence Research Proceedings of the Summer Program.

Rohr, J. J., Itsweire, E. C.,. Helland, K. N., Van Atta,, C. W. (1988), Growth and decay of turbulence in a stably stratified shear flow, J.Fluid Mech.195, 65-77.

Schiestel, R., Elena, L. (1997), Modeling of anisotropic turbulence in rapid rotation, Aerospace Science and Thechnology 7, 441451.

Shih, T.H. (1996), Constitutives relations and realisability of single-point turbulence closures, in turbulence transition modeling, edited by M.Hallback and D.S.Hennigson and A.V.Johansson and P.H.Alfredsson, Dordrecht.

Shih, T., Lumley, L. and Chen, J. (1989), Second order modelling of a passive scalar in a turbulent shear flow, $27^{\text {th }}$ Aerospace Sciences Meeting. Center of turbulent Research, Reno, Nevada.

Speziale, C.G., Sarkar, S., Gatski, T.B. (1990), Modeling the pressure strain correlation of turbulence an invariant dynamical systems approach, NASA Langley research center, Hampton, Virginia 2. 3665-5225.

Thamri Naffouti, L., Lili, T., Bouzaiane, M. (2011), Etude de l'apport du modèle SSG sur la prédiction d'une turbulence stratifiée soumise à un cisaillement non vertical, $20^{\text {ème }}$ Congrès Français de Mécanique.

Zeman, O., Lumley, J.L. (1976), Modeling buoyancy-driven mixed layers, J.Atm.Sci.33, 1974-1988. 